\title{
Multiscale Fluctuation Features of the Dynamic Correlation between Bivariate Time Series
}

\author{
Meihui Jiang, ${ }^{1,2,3}$ Xiangyun Gao, ${ }^{1,2,3}$ Haizhong An, ${ }^{1,2,3}$ Xiaoliang Jia,,2,3 and Xiaoqi Sun ${ }^{1,2,3}$ \\ ${ }^{1}$ School of Humanities and Economic Management, China University of Geosciences, Beijing 100083, China \\ ${ }^{2}$ Key Laboratory of Carrying Capacity Assessment for Resource and Environment, Ministry of Land and Resources, \\ Beijing 100083, China \\ ${ }^{3}$ Open Lab of Talents Evaluation, Ministry of Land and Resources, Beijing 100083, China \\ Correspondence should be addressed to Xiangyun Gao; gxy5669777@126.com
}

Received 1 June 2016; Revised 12 September 2016; Accepted 20 September 2016

Academic Editor: Zhong-Ke Gao

Copyright (C) 2016 Meihui Jiang et al. This is an open access article distributed under the Creative Commons Attribution License, which permits unrestricted use, distribution, and reproduction in any medium, provided the original work is properly cited.

\begin{abstract}
The fluctuation of the dynamic correlation between bivariate time series has some special features on the time-frequency domain. In order to study these fluctuation features, this paper built the dynamic correlation network models using two kinds of time series as sample data. After studying the dynamic correlation networks at different time-scales, we found that the correlation between time series is a dynamic process. The correlation is strong and stable in the long term, but it is weak and unstable in the short and medium term. There are key correlation modes which can effectively indicate the trend of the correlation. The transmission characteristics of correlation modes show that it is easier to judge the trend of the fluctuation of the correlation between time series from the short term to long term. The evolution of media capability of the correlation modes shows that the transmission media in the long term have higher value to predict the trend of correlation. This work does not only propose a new perspective to analyze the correlation between time series but also provide important information for investors and decision makers.
\end{abstract}

\section{Introduction}

A financial time series records the behavior trajectory of a financial market. By analyzing the fluctuation features of time series, we can understand the structures and characteristics of a financial market. There are many studies which focus on the long-term cointegration relationship between two time series [1-4]. However, other scholars have provided evidence that, with the fluctuation of time series, the relationship between any two time series also changes over time [5-7]. The fluctuation of the correlation between time series can help us to detect the dynamic features of the interaction between them.

Since Zhang and Small first proposed that univariate time series can be transformed to a complex network [8], many literatures have proved that different dynamic characteristics of univariate time series show different topological structures [9-11]. In the last decade, the complex network has showed its effectiveness in time series analysis in multiple areas, including financial markets [12], engineering [13-15], medicine [16-18], and geophysics [19]. Based on these existing researches, few studies are concerned with another trigger of turning time series to network: the hidden multiscale information in the dynamic relationship between bivariate time series in the frequency domain. Although some scholars have made great progress in how to derive multifrequency complex network to characterize the dynamical behavior of time series $[20,21]$, it is still a challenge to transfer the dynamic relationship between bivariate time series to a complex network involving multiscale information and to explore the underlying fluctuation features with time and frequency change simultaneously.

Many studies indicate that financial time series contain different information in the time domain and frequency domain and have different fluctuation characteristics in different time-scales [22-25]. The fluctuation in the short term, medium term, and long term can provide different reference information for different purposes [26-28]. To find the multiscale information in the relationship between bivariate time series, the wavelet analysis provides an effective 
solution. The wavelet analysis can obtain the seasonal or periodic characteristics by filtering nonlinear data to satisfy the different investors' needs [29-31]. Thus, it is necessary to decompose the original series using wavelet analysis to obtain the multiscale fluctuation characteristics of time series.

How does one obtain the multiscale dynamic correlation between bivariate time series? As we all know, at each timescale, time series fluctuates over time. If we divide the entire time series into different subperiods, then each subperiod has its own status of the correlation fluctuation. In this study, we defined the correlation modes representing the different correlation statuses [7]. The correlation modes change with time and interact with each other successively, forming the correlation modes transmission networks, which will reveal the fluctuation of the dynamic correlation between the bivariate time series.

In this paper, we design an algorithm to combine the complex network with wavelet analysis to investigate the fluctuation of the dynamic correlation between bivariate time series on the time-frequency domain. First, we decompose the original time series into the decomposed sequences at different time-scales. Next, we build a correlation modes transmission network at each time-scale. Finally, we study the multiscale fluctuation characteristics of the dynamic correlation between bivariate time series using the complex network analytical approach, including the recognition of the key correlation modes, the transmission characteristics, and the transmission media.

\section{Algorithm and Data Description}

2.1. MODWT Wavelet Decomposition. There are various classifications of the discrete wavelet basis used in existing work, such as MODWT (Maximal Overlap Discrete Wavelet Transform), Haar A-Trous, sym4, and db9 wavelets [32-35]. As one of the discrete wavelet transformations, MODWT is widely used [32]. Based on previous studies on the applications of wavelet methods for specific purposes in economics and finance, it appears that MODWT can avoid the adverse effects attributable to the choice of a starting point or an origin for analysis. With help of MODWT, the scale-based analysis of time series can reveal the characteristics of volatility.

Let $\left\{X_{t}, t=0,1,2, \ldots, N-1\right\}$ be a time series, where $N$ is the length of time series. The MODWT of level $J_{0}$ for the original time series $X_{t}$ yields the column vectors $\widetilde{D_{1}}, \widetilde{D_{2}}, \ldots, \widetilde{D_{j_{0}}}$ and $\widetilde{S_{j_{0}}}$ for any positive integer $J_{0}$, each of which has $N$ dimensions. $\widetilde{D_{j}}$ contains the MODWT wavelet coefficients associated with changes in $X_{t}$ between scale $2^{j-1}$ and scale $2^{j}$, and $\widetilde{S_{j}}$ contains the MODWT scaling coefficients associated with the smoothness of $X_{t}$ at the scale $2^{j_{0}}$.

According to the output from the filters at each scale, the time series $X_{t}$ can be decomposed and reconstructed into wavelet details and approximation as follows:

$$
X_{t}=\sum_{j=1}^{J_{0}} \widetilde{D_{j}}+\widetilde{S_{j_{0}}}
$$

Wavelet decomposition based on $n$ time-scales $(n=$ $1,2, \ldots$ ) includes $D_{1}, D_{2}, \ldots, D_{n}$, representing different deviation subseries from the trend, and $S_{n}$, representing the longterm trend subseries:

$$
\begin{aligned}
\text { Original time series }= & S_{n}+D_{n}+D_{n-1}+\cdots+D_{2} \\
& +D_{1} .
\end{aligned}
$$

2.2. The Multiscale Correlation Modes Transmission Networks Construction. After decomposing time series using MODWT, we obtained the subseries of the time series at different time-scales. At each time-scale, we divide the subseries into different subperiods. In this paper, we use sliding windows. Compared with dividing time series into different individual time periods, the advantage of sliding windows is that they contain the features of memory and transitivity $[6,36-38]$. The length of sliding windows depends on the needs of the analysis. If the goal is to study the short-term fluctuation of time series, the length can be set to a smaller value. If the goal is to understand the fluctuation of time series in the long term, the length can be set to a larger value.

In this paper, we set the size of a sliding window for 10 days because we want to analyze the short-term fluctuation features of the correlation between bivariate time series. First, we choose day $t$ as start point and get subperiod $d_{t}$ which is from day $t$ to day $t+9$. Then, we choose day $t+1$ as start point and get subperiod $d_{t+1}$ which is from day $t+1$ to day $t+10$. By that analogy, we can obtain a series of subperiods. Then, at each time-scale, we follow four steps to construct the correlation modes transmission networks.

Step 1. We calculate the correlation coefficient between two time series in each subperiod at the same time-scale and then obtain a sequence of the correlation coefficients. The sequence shows the fluctuation of the correlation between bivariate time series at each time-scale [7]. In this paper, we use the Pearson Correlation Coefficient to measure the correlation between time series as follows:

$$
r_{x y}=\frac{\sum_{i=1}^{n}\left(x_{i}-\bar{x}\right)\left(y_{i}-\bar{y}\right)}{\sqrt{\sum_{i=1}^{n}\left(x_{i}-\bar{x}\right)^{2}} \sqrt{\sum_{i=1}^{n}\left(y_{i}-\bar{y}\right)^{2}}} .
$$

$x$ and $y$ denote the value of the subseries of two time series at each time-scale, where $\bar{x}$ and $\bar{y}$ denote the mean of the subseries. $x_{i}$ and $y_{i}$ denote the value of the subseries at time $i$. Let $n$ denote the values' number of the subseries. The range of the value of $r_{x y}$ is $[-1,1]$.

Step 2. We symbolize the strength of the correlation between two time series at different time-scales. After calculating the correlation coefficient between two time series at each time-scale, we divide the correlation coefficient into 5 levels. Then, we get a sequence of symbolized correlation coefficients 
at each time-scale. The 5 levels are defined as follows [36]:

$$
\begin{aligned}
& \mathrm{CS}_{i} \\
& =\left\{\begin{array}{l}
L_{1}\left(-1 \leq r_{x y} \leq-0.8, \text { strongly negative correlated }\right) \\
L_{2}\left(-0.8<r_{x y} \leq-0.3 \text {, weakly negative correlated }\right) \\
L_{3}\left(-0.3<r_{x y}<0.3 \text {, no correlation }\right) \\
L_{4}\left(0.3 \leq r_{x y}<0.8, \text { weakly positive correlated }\right) \\
L_{5}\left(0.8 \leq r_{x y} \leq 1, \text { strongly positive correlated }\right) .
\end{array}\right.
\end{aligned}
$$

Step 3. We define the correlation modes between two time series by the coarse graining process [39]. The coarse graining process is the concept in the phase space. The smallest grain in the phase space is one dot. If we consider roughly a set of dots as a mode, the study of a series of the dots can become the research on modes consisting of a complex system [36, 40]. Similar to the process for dividing the time series into different subperiods, we use sliding windows to obtain a series of the correlation modes from the symbolized correlation coefficients. We set the size of a sliding window for a transaction period ( 5 days). Thus, the correlation modes are comprised of 5 symbolized correlation coefficients. Each correlation mode represents the status of the correlation fluctuation of two time series at the corresponding time-scale.

Step 4. We build the correlation modes transmission networks at different time-scales. Like we defined at Step 3, we obtain a series of correlation modes with the moving of the sliding window. One correlation mode converted to another as time goes by: mode $_{1} \rightarrow$ mode $_{2} \rightarrow$ mode $_{3} \rightarrow$ $\cdots \rightarrow$ mode $_{n}$. Because the conversion between two types of correlation modes would repeat in the transmission process, the trajectory of the conversion among correlation modes forms a network. In the correlation modes transmission network, we take the correlation modes as nodes and the succeeding sequence relations between the correlation modes as edges. The weight of an edge is the frequency of the transformation between two types of correlation modes. The process of building the correlation modes transmission networks is shown in Figure 1.

2.3. Data Description. In this paper, we focus on the relationship between bivariate time series. As the precondition of our work, there should be the theoretical and realistic relationship between two time series. Based on this, we choose the international crude oil spot market and natural gas spot market as the sample markets. The data we choose are the WTI crude oil spot price from NYMEX and natural gas spot price from the Henry Hub. The data cover the period from January 7, 1997, to November 23, 2015. All data are selected from EIA (US Energy Information Administration).

$P_{t}$ denotes the daily closing price on day $t$. The daily price return is calculated as follows:

$$
r_{t}=\ln \left(P_{t}\right)-\ln \left(P_{t-1}\right)
$$

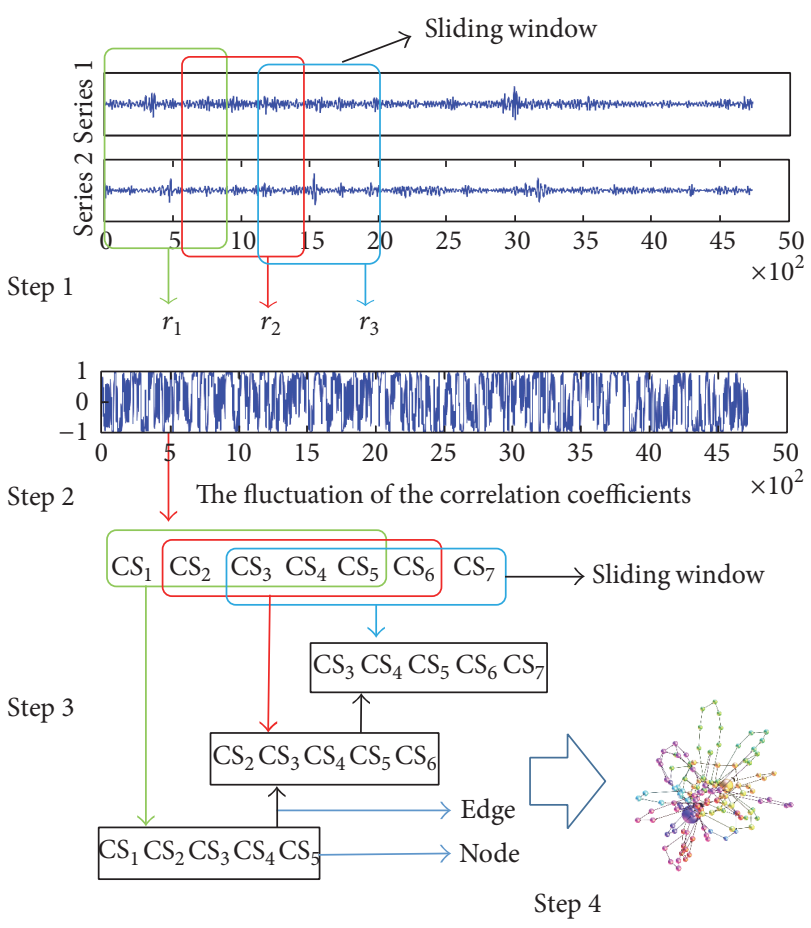

FIGURE 1: Schematic illustration of building the correlation modes transmission networks. Step 1: calculate the correlation coefficient in each subperiod. Step 2: symbolize the strength of the correlation coefficients. Step 3: abstract the correlation modes from symbolized correlation coefficients. Step 4: build the correlation modes transmission networks.

\section{Empirical Results}

3.1. MODWT Wavelet Decomposition for the Return Series of Crude Oil and Natural Gas Prices. To study the multiscale phenomenon in the fluctuation of the correlation between the daily crude oil spot price and natural gas spot price, we decompose the return series of original prices into different subseries. We choose $J_{0}=6$ as the maximum level of MODWT. Many researches show that using 6 levels can achieve an optimal balance between sample size and the length of the filter for financial or economic time series $[41,42]$. In our research, decomposing the return series into 6 levels can divide the time series into three fluctuation periods: short term, medium term, and long term, so the results can provide targeted suggestion for different investors and decision makers. The scale $D_{1}$ represents the 2-4 days' horizon and the scale $D_{2}$ represents the $4-8$ days' horizon. These two scales represent the short-term variation. The scales $D_{3}(8-$ 16 days) and $D_{4}$ (16-32 days) represent the medium-term variation. The scales $D_{5}$ (32-64 days) and $D_{6}$ (64-128 days) represent the long-term variation. $S_{6}$ represents the trend of the original series obtained after subtracting $D_{1}, D_{2}, D_{3}$, $D_{4}, D_{5}$, and $D_{6}$ from the series. The results of the wavelet decomposition are shown in Figures 2 and 3.

3.2. The Statistic Characteristics of the Correlation. After decomposing the return series of crude oil and natural gas 


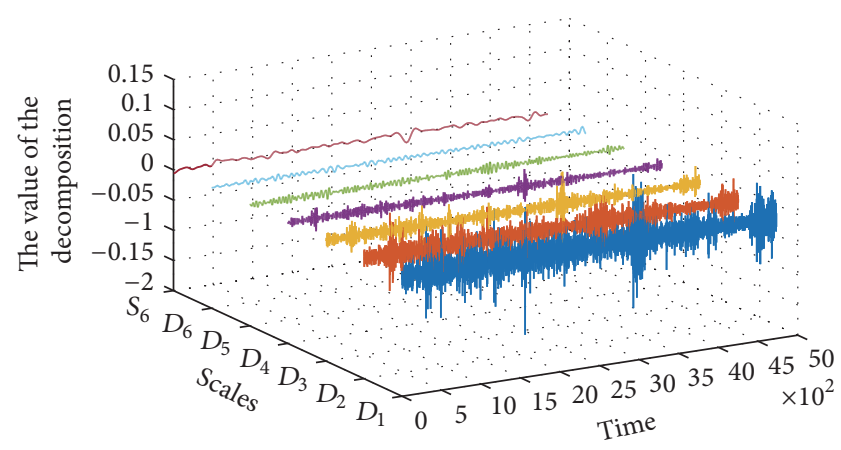

FIGURE 2: The wavelet decomposition for the return series of crude oil price.

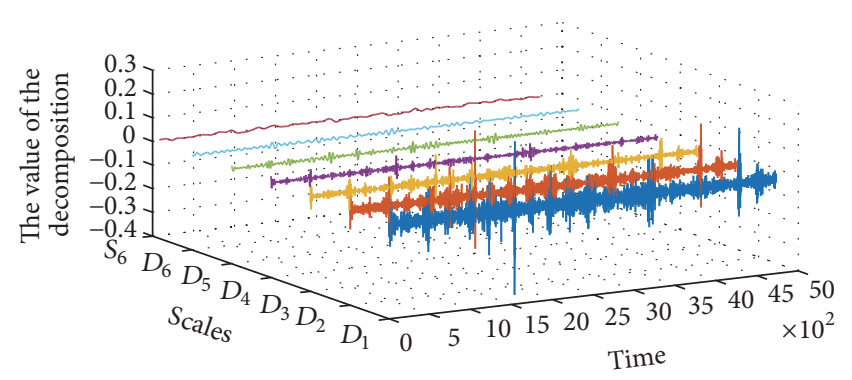

FIGURE 3: The wavelet decomposition for the return series of natural gas price.

prices, we use sliding windows to calculate the dynamic correlation coefficients between crude oil and natural gas prices at each time-scale (see Figure 4). According to Figure 4, the correlation coefficients change over time. The fluctuation of the correlation has the multiscale feature. For the scales $D_{1}$ and $D_{2}$, in the short term, the correlation between crude oil and natural gas prices strongly fluctuates with small change but high frequency. For the scales $D_{3}$ and $D_{4}$, in the medium term, the change ranges are larger but the frequency is lower. For the long-term $D_{5}$ and $D_{6}$, the correlation has the largest change ranges and lowest frequency. Furthermore, the correlation coefficients tend to -1 and 1 and usually last for a period of time after peaking in the long term. It means that the correlation between crude oil and natural gas prices gradually strengthens and tends to be stable with the increase of the time-scales.

After symbolizing the correlation coefficients, the correlation coefficients are divided into 5 levels which are strongly negatively correlated, weakly negatively correlated, not correlated, weakly positively correlated, and strongly positively correlated. The distribution of the correlation levels at different time-scales over time is shown in Figure 5. The five colors from dark to light represent five levels of the correlation. According to Figure 5, with the increase of the time-scales, the percentage accounted for by the three colors between the darkest and the lightest continues to decrease, but the percentage accounted for by the darkest and the lightest continues to increase. After calculating, the percentage of the strongly correlation for the scales $D_{1}$ and $D_{2}$, respectively, accounts for $3.3 \%$ and $8.2 \%$. For the scales $D_{3}$
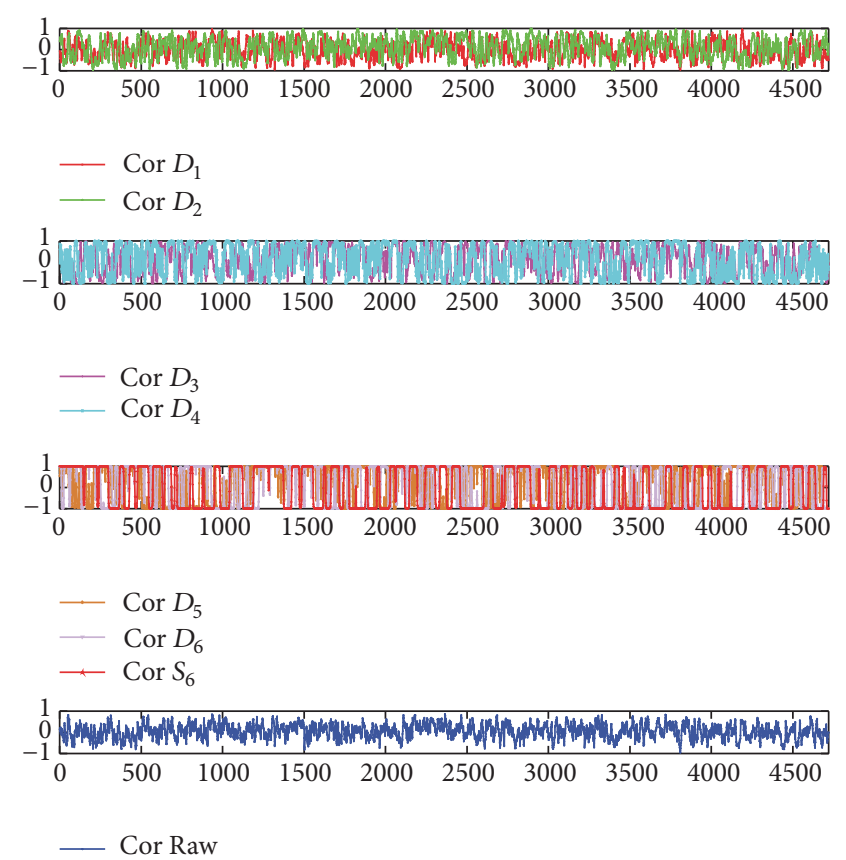

Figure 4: The fluctuation of the correlation between crude oil and natural gas prices at different time-scales.

and $D_{4}$, the percentage, respectively, increases to $26.4 \%$ and $47.1 \%$. For the scales $D_{5}$ and $D_{6}$, the percentage is relatively high and it, respectively, accounts for $73 \%$ and $82.7 \%$. The same color continues longer with the increase of the timescales. This result also means that the correlation between crude oil and natural gas prices gradually strengthens and tends to be stable with the increase of the time-scales from another perspective.

3.3. The Recognition of the Key Correlation Modes. To recognize the key correlation modes at different time-scales, we choose weighted out-degree to measure the importance of the correlation modes. The weighted out-degree of one node represents that node's weight connecting to adjacent nodes. The weighted out-degree is calculated as follows:

$$
w_{i}^{\text {out }}=\sum_{j \in N_{i}} w_{i, j}
$$

$N_{i}$ denotes the set of the nodes connecting to node $i . w_{i, j}$ denotes the weight of the edge from node $i$ to node $j$.

The weighted out-degree at all time-scales follows the power-law distribution (Figure 6). The values of weighted out-degree of most correlation modes are small but few modes' values of weighted out-degree are very large. This finding means that there are few modes that have a strong effect on the transmission process of the correlation modes.

Figure 7 shows the distributions of weighted out-degree at all time-scales during the time span. In Figure 7, different colors represent different value of weighted out-degree. The lighter the color is, the larger the value of weighted outdegree is. Every value of weighted out-degree represents a correlation mode, so every color represents a correlation 


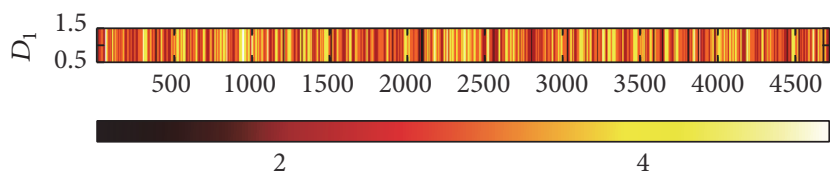

$\stackrel{N}{ }^{2} \begin{array}{ll}1.5 \\ 0.5\end{array}$ $\begin{array}{lllllllll}500 & 1000 & 1500 & 2000 & 2500 & 3000 & 3500 & 4000 & 4500\end{array}$

2

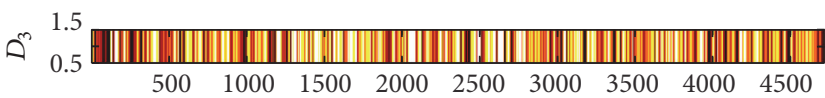

2

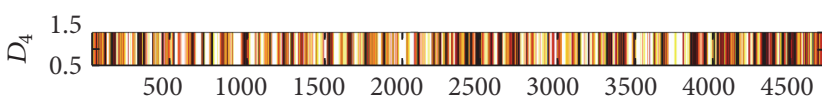

2

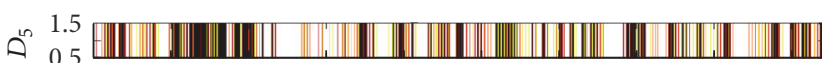

$\begin{array}{lllllllll}500 & 1000 & 1500 & 2000 & 2500 & 3000 & 3500 & 4000 & 4500\end{array}$

24

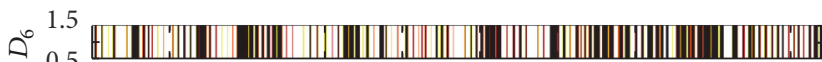

$\begin{array}{lllllllll}500 & 1000 & 1500 & 2000 & 2500 & 3000 & 3500 & 4000 & 4500\end{array}$

2

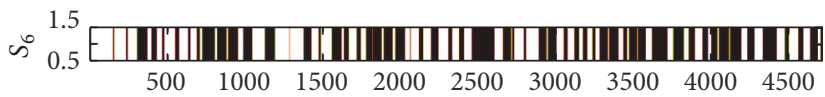

FIGURE 5: The distribution of the symbolized correlation coefficients over time at different time-scales.

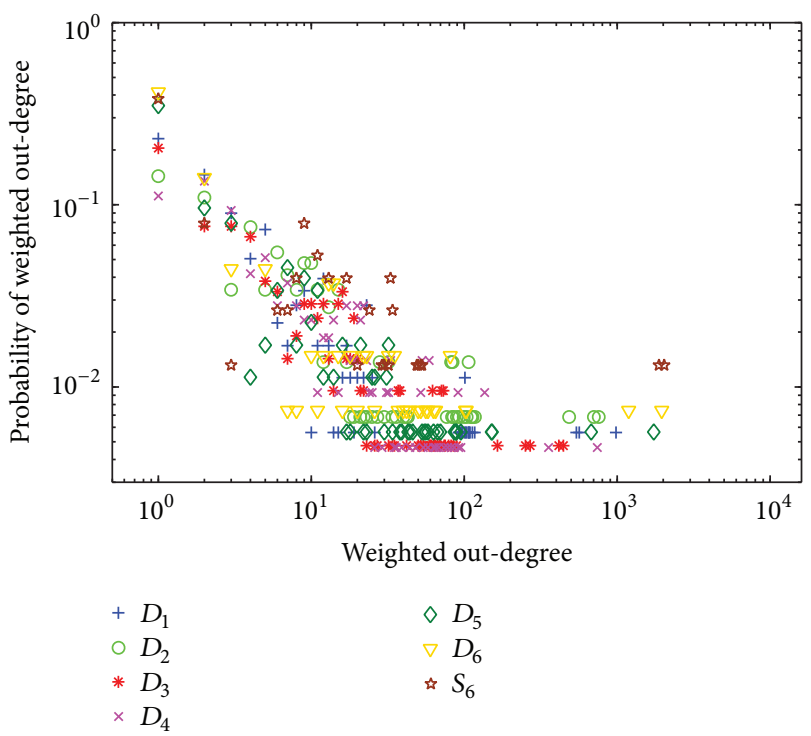

Figure 6: The distribution of weighted out-degree.

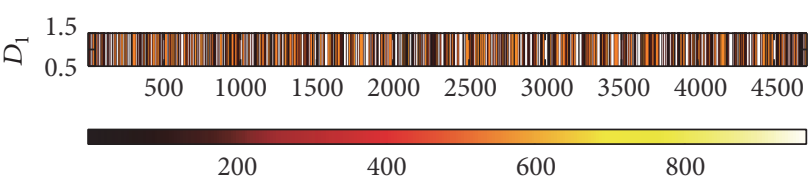

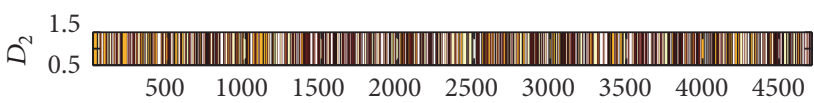

$200 \quad 600$

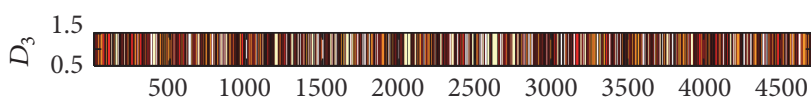

200

400

А́

$\begin{array}{lllllllll}500 & 1000 & 1500 & 2000 & 2500 & 3000 & 3500 & 4000 & 4500\end{array}$

$\begin{array}{lll}\square & 400 & 600\end{array}$

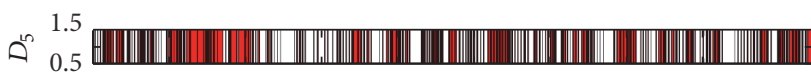
$\begin{array}{lllllllll}500 & 1000 & 1500 & 2000 & 2500 & 3000 & 3500 & 4000 & 4500\end{array}$

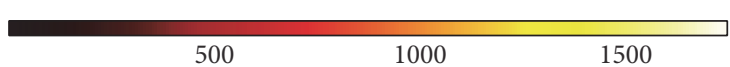

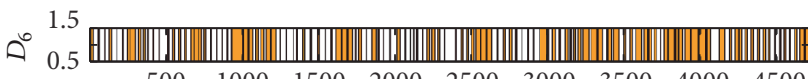

$\begin{array}{lllllllll}500 & 1000 & 1500 & 2000 & 2500 & 3000 & 3500 & 4000 & 4500\end{array}$

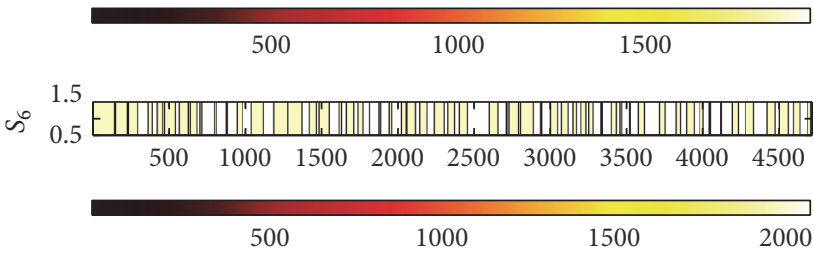

FIGURE 7: The distribution of weighted out-degree on the time span.

mode. The lighter the color is, the more important the correlation modes are. According to Figure 7, we can find that the key correlation modes have more and more important influence on the transmission process with the increase of the time-scales. The width of one color bar represents the time a correlation mode continues. Each mode will continue for some time and then convert into another mode. The average time that the correlation modes at each time-scale continue is shown in Table 1 . In the short term, $D_{1}$ and $D_{2}$ (2-8 days), and in the medium term, $D_{3}$ and $D_{4}$ (8-32 days), the color distributions of weighted out-degree are uneven, and the colors all continue for a short time. It means that the transmission speed of the correlation modes is fast. The process that one mode converts to another has stochastic characteristics. In the long term, $D_{5}$ and $D_{6}$ (32-128 days), the proportion of the light color increases and the colors continue longer. It means that the transmission speed of the correlation modes slows with the increase of the time-scales.

Ranking the correlation modes at all time-scales by weighted out-degree, we can recognize the key correlation modes for the dynamic process. The key correlation modes for the correlation modes transmission networks at different 
TABLE 1: The average time that the correlation modes at all timescales continue.

\begin{tabular}{lc}
\hline Time-scale & The average time (days) \\
\hline$D_{1}$ & 1.64 \\
$D_{2}$ & 1.67 \\
$D_{3}$ & 1.39 \\
$D_{4}$ & 1.31 \\
$D_{5}$ & 1.97 \\
$D_{6}$ & 2.94 \\
$S_{6}$ & 6.07 \\
\hline
\end{tabular}

Note: the average time $=$ the total time/the number of the color bars ranking by time.

TABLE 2: The key correlation modes at all time-scales.

\begin{tabular}{|c|c|c|}
\hline Time-scale & Mode & Percentage \\
\hline \multirow{3}{*}{$D_{1}$} & $L_{3} L_{3} L_{3} L_{3} L_{3}$ & $10.4 \%$ \\
\hline & $L_{2} L_{2} L_{2} L_{2} L_{2}$ & $6.0 \%$ \\
\hline & $L_{4} L_{4} L_{4} L_{4} L_{4}$ & $5.7 \%$ \\
\hline \multirow{3}{*}{$D_{2}$} & $L_{3} L_{3} L_{3} L_{3} L_{3}$ & $8.0 \%$ \\
\hline & $L_{4} L_{4} L_{4} L_{4} L_{4}$ & $7.5 \%$ \\
\hline & $L_{2} L_{2} L_{2} L_{2} L_{2}$ & $5.1 \%$ \\
\hline \multirow{3}{*}{$D_{3}$} & $L_{4} L_{4} L_{4} L_{4} L_{4}$ & $4.7 \%$ \\
\hline & $L_{5} L_{5} L_{5} L_{5} L_{5}$ & $4.4 \%$ \\
\hline & $L_{3} L_{3} L_{3} L_{3} L_{3}$ & $2.9 \%$ \\
\hline \multirow{3}{*}{$D_{4}$} & $L_{5} L_{5} L_{5} L_{5} L_{5}$ & $7.9 \%$ \\
\hline & $L_{1} L_{1} L_{1} L_{1} L_{1}$ & $3.8 \%$ \\
\hline & $L_{4} L_{5} L_{5} L_{5} L_{5}$ & $1.4 \%$ \\
\hline \multirow{3}{*}{$D_{5}$} & $L_{5} L_{5} L_{5} L_{5} L_{5}$ & $18.4 \%$ \\
\hline & $L_{1} L_{1} L_{1} L_{1} L_{1}$ & $7.2 \%$ \\
\hline & $L_{5} L_{5} L_{5} L_{5} L_{4}$ & $1.6 \%$ \\
\hline \multirow{3}{*}{$D_{6}$} & $L_{5} L_{5} L_{5} L_{5} L_{5}$ & $20.7 \%$ \\
\hline & $L_{1} L_{1} L_{1} L_{1} L_{1}$ & $12.6 \%$ \\
\hline & $L_{5} L_{5} L_{5} L_{5} L_{4}$ & $1.1 \%$ \\
\hline \multirow{3}{*}{$S_{6}$} & $L_{1} L_{1} L_{1} L_{1} L_{1}$ & $21.5 \%$ \\
\hline & $L_{5} L_{5} L_{5} L_{5} L_{5}$ & $19.9 \%$ \\
\hline & $L_{2} L_{1} L_{1} L_{1} L_{1}$ & $0.6 \%$ \\
\hline
\end{tabular}

time-scales are shown in Table 2. At the time-scales $D_{1}$ and $D_{2}$ (2-8 days), the key mode is $L_{3} L_{3} L_{3} L_{3} L_{3}$. At the timescales $D_{3}$ and $D_{4}$ (8-32 days), the key mode is $L_{4} L_{4} L_{4} L_{4} L_{4}$. At the time-scales $D_{5}$ and $D_{6}$ (32-128 days), it is $L_{5} L_{5} L_{5} L_{5} L_{5}$. Thus, crude oil and natural gas prices are primarily not correlated or are weakly correlated in the short term (2-8 days) and medium term (8-32 days). In the long term (32-128 days), crude oil and natural gas prices are primarily strongly positively correlated.

\subsection{The Transmission Characteristics of the Correlation Modes.} The transmission characteristics of the correlation modes include two parts: the transmission speed and the transmission direction. We choose the average path length to measure the transmission speed. The transmission direction is

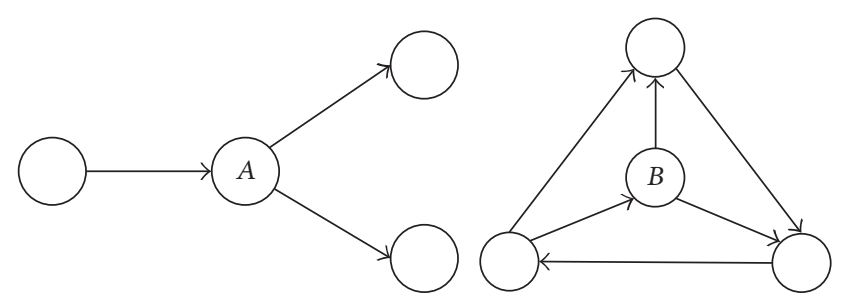

FIGURE 8: The principle of the weighted clustering coefficient. Note: $C_{A}^{w}=0, C_{B}^{w}=1$.

described by the average weighted clustering coefficient. The average path length equals the average number of the edges that one correlation mode will pass to reach another. If one correlation mode will pass few correlation modes to another correlation mode, it means that the transmission speed is fast. The average weighted clustering coefficient represents the tightness of the network. The larger the average weighted clustering coefficient is, the more closely the correlation modes connect each other. In the loose network, the paths formed by the transmission of the correlation modes are mostly catenarian. In the tight network, the transmission of the correlation modes is more random. The principle of the weighted clustering coefficient is shown in Figure 8.

The average path length is calculated as follows:

$$
L=\frac{1}{N(N-1)} \sum_{i \neq j} l_{i, j}
$$

$N$ denotes the number of the nodes in the network and $l_{i, j}$ denotes the shortest path from node $i$ to node $j$.

The weighted clustering coefficient of node $i$ is calculated as follows [43]:

$$
C_{i}^{w}=\frac{1}{w_{i}\left(k_{i}-1\right)} \sum_{j, h} \frac{\left(w_{i j}+w_{i h}\right)}{2} a_{i j} a_{i h} a_{j h} .
$$

$w_{i}$ is the weighted degree of node $i . k_{i}$ is the degree of node $i . w_{i j}$ is the weight of the edge connecting node $i$ to node $j$. $a_{i j}$ is the adjacent matrix of node $i$ and node $j$. The mean of the weighted clustering coefficients of all nodes is the average weighted clustering coefficient of the network.

According to Figure 9, the general trend is that the average path length increases and the average weighted clustering coefficient decreases with the increase of the time-scales. This result means that transmission speed becomes slower with the increase of the time-scales. In the short term and medium term, the transmission of the correlation modes has the stochastic features. In the long term, the transmission of the correlation modes has the characteristics that have a certain direction.

3.5. The Recognition of the Transmission Media. In the transmission of the correlation modes, some correlation modes connect two or more correlation modes which do not have a direct connection, and these modes are called the transmission media. Recognizing the important transmission 


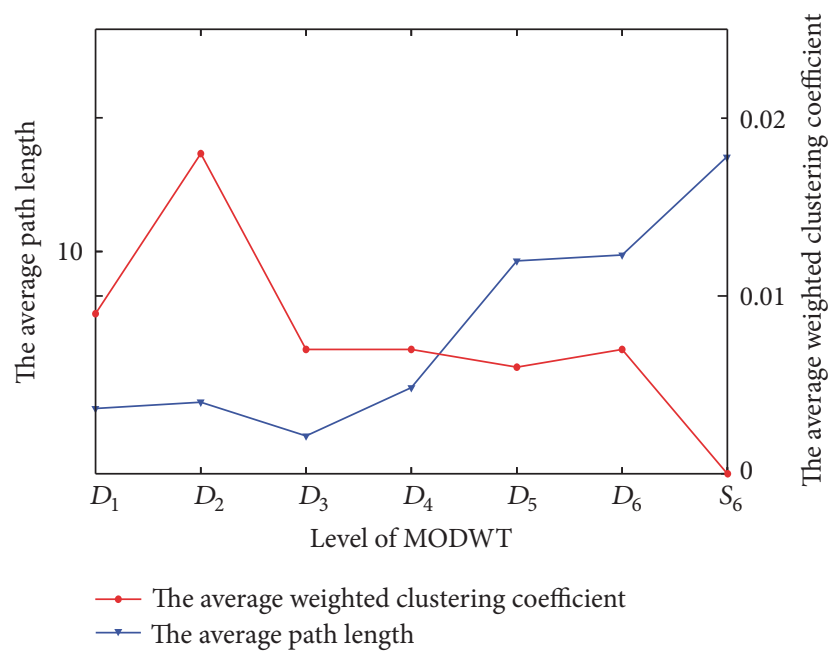

FIgURE 9: The evolution of the transmission characteristics of the networks.

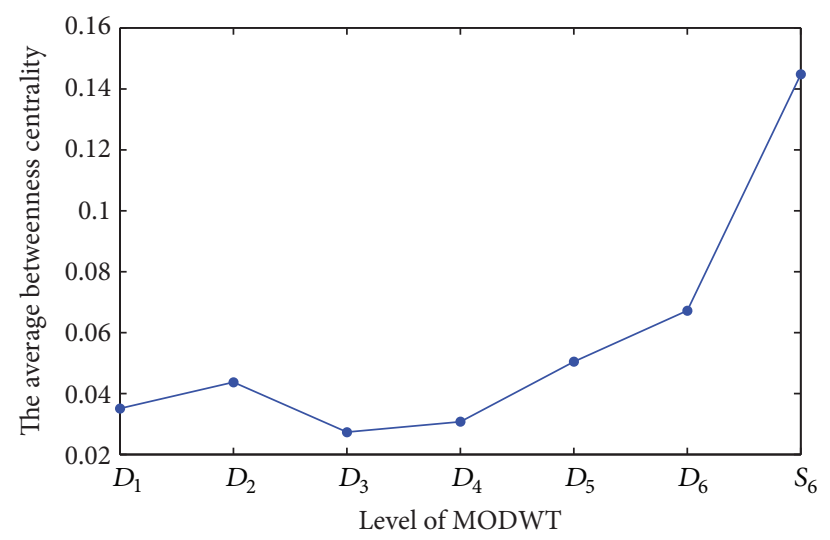

Figure 10: The evolution of the media capability on the networks.

media can help us to judge the tendency of the correlation between crude oil and natural gas prices. In this paper, we choose the betweenness centrality to measure the media capability of the correlation modes in the correlation modes transmission networks at different time-scales. The mean of the betweenness centrality of all correlation modes is the average betweenness centrality of the network, which represents the average level of the media capability that the modes have in the networks. The standard betweenness centrality is calculated as follows [5]:

$$
\mathrm{BC}_{i}=\frac{\sum_{j}^{n} \sum_{k}^{n} g_{j k}(i) / g_{j k}}{n^{2}-3 n+2}, \quad j \neq k \neq i, \quad j<k
$$

$g_{j k}(i)$ denotes the number of the shortest paths that pass node $i$ between node $j$ and node $k . g_{j k}$ denotes the number of the shortest paths between node $j$ and node $k$.

Figure 10 shows that the average betweenness centrality is stable in the short term and medium term, and the average betweenness centrality in the long term is clearly higher. Therefore, the modes have stronger media capability in the
TABLE 3: The key transmission media at different time-scales.

\begin{tabular}{lcc}
\hline Time-scale & Mode & Betweenness centrality \\
\hline \multirow{3}{*}{$D_{1}$} & $L_{4} L_{4} L_{3} L_{3} L_{3}$ & 0.138794 \\
& $L_{3} L_{3} L_{4} L_{4} L_{4}$ & 0.121528 \\
& $L_{2} L_{2} L_{3} L_{4} L_{4}$ & 0.112671 \\
\hline \multirow{2}{*}{$D_{2}$} & $L_{3} L_{3} L_{4} L_{4} L_{4}$ & 0.163039 \\
& $L_{3} L_{2} L_{2} L_{2} L_{2}$ & 0.122893 \\
& $L_{2} L_{2} L_{3} L_{3} L_{3}$ & 0.118542 \\
\hline \multirow{3}{*}{$D_{3}$} & $L_{2} L_{2} L_{3} L_{4} L_{4}$ & 0.122249 \\
& $L_{4} L_{4} L_{3} L_{3} L_{2}$ & 0.087785 \\
& $L_{4} L_{3} L_{2} L_{2} L_{3}$ & 0.079774 \\
\hline \multirow{3}{*}{$D_{4}$} & $L_{4} L_{5} L_{5} L_{5} L_{4}$ & 0.147686 \\
& $L_{5} L_{5} L_{5} L_{4} L_{3}$ & 0.133837 \\
& $L_{2} L_{3} L_{4} L_{5} L_{5}$ & 0.116286 \\
\hline \multirow{3}{*}{$D_{5}$} & $L_{1} L_{1} L_{1} L_{2} L_{3}$ & 0.276475 \\
& $L_{1} L_{1} L_{1} L_{1} L_{2}$ & 0.266845 \\
& $L_{5} L_{5} L_{5} L_{4} L_{3}$ & 0.255829 \\
\hline \multirow{3}{*}{$D_{6}$} & $L_{5} L_{5} L_{5} L_{5} L_{4}$ & 0.520667 \\
& $L_{4} L_{5} L_{5} L_{5} L_{5}$ & 0.520667 \\
& $L_{1} L_{1} L_{1} L_{1} L_{2}$ & 0.40357 \\
\hline \multirow{3}{*}{$S_{6}$} & $L_{2} L_{1} L_{1} L_{1} L_{1}$ & 0.549309 \\
& $L_{1} L_{1} L_{1} L_{1} L_{1}$ & 0.549309 \\
& $L_{1} L_{1} L_{1} L_{1} L_{2}$ & 0.549309 \\
\hline
\end{tabular}

long term, and it explains that the transmission of the correlation modes has the characteristics that have a certain direction from another perspective.

There are some key transmission media in the correlation modes transmission networks at different time-scales, shown in Table 3. When these modes appear in the transmission, they usually predict that the correlation between crude oil and natural gas prices is in the midst of transformation.

\section{Conclusion}

For studying the multiscale fluctuation features of the dynamic correlation between bivariate time series, we proposed an algorithm to map the fluctuation of the correlation into networks which can show us the nonlinear and nonstationary moving trajectory of the correlation modes. After analyzing the structure characteristics of the correlation modes transmission networks at different time-scales, we got some findings that can help us understand the fluctuation characteristics of time series on both the time domain and frequency domain.

The correlation between bivariate time series is in the fluctuation, which means that the correlation is dynamic. The fluctuation of the correlation at different time-scales has different features. In the short term, the correlation between crude oil and natural gas prices strongly fluctuates with small change ranges but high frequency. In the long term, the change ranges are large but the frequency is low. There are few key correlation modes which show the main status of the correlation at different time-scales. In the short term, medium term, and long term, the key correlation modes are, 
respectively, $L_{3} L_{3} L_{3} L_{3} L_{3}, L_{4} L_{4} L_{4} L_{4} L_{4}$, and $L_{5} L_{5} L_{5} L_{5} L_{5}$. Different transmission characteristics exist in the dynamic process of the correlation between bivariate time series at different time-scales. With an increase of the time-scales, the transmission speed continues decreasing, and the transmission among the correlation modes becomes more directional. This result means that it is easier to judge the trend of the fluctuation of the correlation between bivariate time series in the long term. For different time-scales from $D_{1}$ (2-4 days) to $D_{6}$ (64-128 days), the correlation mode that has the strongest media capability is $L_{4} L_{4} L_{3} L_{3} L_{3}, L_{3} L_{3} L_{4} L_{4} L_{4}, L_{2} L_{2} L_{3} L_{4} L_{4}$, $L_{4} L_{5} L_{5} L_{5} L_{4}, L_{1} L_{1} L_{1} L_{2} L_{3}$, and $L_{5} L_{5} L_{5} L_{5} L_{4}$.

These results show that the relationship between crude oil market and natural gas market changes over time and the fluctuation of the relationship is not random. Thus, there exist some rules in the fluctuation of relationship between crude oil market and natural gas market that we can discover for providing the suggestion for the investors and decision makers. For example, due to the dynamic correlation, the decision or investment strategy concerning crude oil and natural gas markets should also be dynamic and properly adjusted according to reality. The key correlation modes can provide information for adjustment of the strategy because they effectively indicate the trend of the dynamic correlation and provide predicting signal of the energy market for decision makers. The transmission characteristics show that it is easier and easier to judge the trend of the fluctuation of the correlation between crude oil and natural gas prices from the short term to the long term. Thus, for the decision makers and the investors, predictions may be more accurate for natural gas (crude oil) prices depend on crude oil (natural gas) prices in the long term. The correlation modes which have stronger media capability have higher reference value to predict the trend of correlation with the increase of time-scales.

In this paper, we uncover the dynamic process of the correlation between bivariate time series at different timescales. Furthermore, we will consider more time series in our model so that we can study the entire financial market.

\section{Competing Interests}

The authors declare that they have no competing interests.

\section{Acknowledgments}

This research is supported by grants from the National Natural Science Foundation of China (Grant no. 71173199), the China Scholarship Council (File no. 201606405009), the Fundamental Research Funds for the Central Universities (Grant no. 2-9-2015-303) and the fund from Key Laboratory of Carrying Capacity Assessment for Resource and Environment, Ministry of Land and Resources (Grant no. CCA2016.10). The authors are grateful for Shupei Huang, who suggests significant improvements to the manuscript.

\section{References}

[1] P. M. Robinson, "Semiparametric analysis of long-memory time series," The Annals of Statistics, vol. 22, no. 1, pp. 515-539, 1994.
[2] P. C. Phillips and H. R. Moon, "Linear regression limit theory for nonstationary panel data," Econometrica, vol. 67, no. 5, pp. 1057-1111, 1999.

[3] K. Prakash and D. Maiti, "Does devaluation improve trade balance in small island economies? The case of Fiji," Economic Modelling, vol. 55, pp. 382-393, 2016.

[4] J. I. Miller, "Conditionally efficient estimation of long-run relationships using mixed-frequency time series," Econometric Reviews, vol. 35, no. 6, pp. 1142-1171, 2016.

[5] X. Gao, H. An, W. Fang, H. Li, and X. Sun, “The transmission of fluctuant patterns of the forex burden based on international crude oil prices," Energy, vol. 73, pp. 380-386, 2014.

[6] X. Gao, H. An, W. Fang, X. Huang, H. Li, and W. Zhong, "Characteristics of the transmission of autoregressive sub-patterns in financial time series," Scientific Reports, vol. 4, article 6290, 2014.

[7] X. Huang, H. Z. An, X. Y. Gao, X. Q. Hao, and P. P. Liu, "Multiresolution transmission of the correlation modes between bivariate time series based on complex network theory," Physica A: Statistical Mechanics and Its Applications, vol. 428, pp. 493506, 2015.

[8] J. Zhang and M. Small, "Complex network from pseudoperiodic time series: topology versus dynamics," Physical Review Letters, vol. 96, no. 23, Article ID 238701, 2006.

[9] L. Lacasa, B. Luque, F. Ballesteros, J. Luque, and J. C. Nuño, "From time series to complex networks: the visibility graph," Proceedings of the National Academy of Sciences of the United States of America, vol. 105, no. 13, pp. 4972-4975, 2008.

[10] X. Xu, J. Zhang, and M. Small, "Superfamily phenomena and motifs of networks induced from time series," Proceedings of the National Academy of Sciences of the United States of America, vol. 105, no. 50, pp. 19601-19605, 2008.

[11] Z.-K. Gao, P.-C. Fang, M.-S. Ding, and N.-D. Jin, "Multivariate weighted complex network analysis for characterizing nonlinear dynamic behavior in two-phase flow," Experimental Thermal and Fluid Science, vol. 60, pp. 157-164, 2015.

[12] X. Gao, H. An, W. Fang et al., "Transmission of linear regression patterns between time series: from relationship in time series to complex networks," Physical Review E-Statistical, Nonlinear, and Soft Matter Physics, vol. 90, no. 1, Article ID 012818, 2014.

[13] Z.-K. Gao, X.-W. Zhang, N.-D. Jin, R. V. Donner, N. Marwan, and J. Kurths, "Recurrence networks from multivariate signals for uncovering dynamic transitions of horizontal oil-water stratified flows," Europhysics Letters, vol. 103, no. 5, Article ID 50004, 2013.

[14] Z.-K. Gao and N.-D. Jin, "A directed weighted complex network for characterizing chaotic dynamics from time series," Nonlinear Analysis: Real World Applications, vol. 13, no. 2, pp. 947-952, 2012.

[15] Z. K. Gao, Y. X. Yang, L. S. Zhai, N. D. Jin, and G. R. Chen, "A four-sector conductance method for measuring and characterizing low-velocity oil-water two-phase flows," IEEE Transactions on Instrumentation and Measurement, vol. 65, no. 7, pp. 1690-1697, 2016.

[16] K. Lehnertz, G. Ansmann, S. Bialonski, H. Dickten, C. Geier, and S. Porz, "Evolving networks in the human epileptic brain," Physica D. Nonlinear Phenomena, vol. 267, pp. 7-15, 2014.

[17] E. Siggiridou, D. Kugiumtzis, and V. K. Kimiskidis, "Correlation networks for identifying changes in brain connectivity during epileptiform discharges and transcranial magnetic stimulation," Sensors, vol. 14, no. 7, pp. 12585-12597, 2014. 
[18] M. Zanin, P. Sousa, D. Papo et al., "Optimizing functional network representation of multivariate time series," Scientific Reports, vol. 2, article 630, 2012.

[19] M. Paluš, D. Hartman, J. Hlinka, and M. Vejmelka, "Discerning connectivity from dynamics in climate networks," Nonlinear Processes in Geophysics, vol. 18, no. 5, pp. 751-763, 2011.

[20] Z.-K. Gao, Y.-X. Yang, P.-C. Fang, N.-D. Jin, C.-Y. Xia, and L.D. Hu, "Multi-frequency complex network from time series for uncovering oil-water flow structure," Scientific Reports, vol. 5, article 8222, 2015.

[21] Z.-K. Gao, Y.-X. Yang, P.-C. Fang, Y. Zou, C.-Y. Xia, and M. $\mathrm{Du}$, "Multiscale complex network for analyzing experimental multivariate time series," EPL, vol. 109, no. 3, Article ID 30005, 2015.

[22] J. Kabanda, O.-S. Kwon, and G. Kwon, "Time and frequency domain analyses of the Hualien Large-Scale seismic test," Nuclear Engineering and Design, vol. 295, pp. 261-275, 2015.

[23] M. G. De Giorgi, S. Campilongo, A. Ficarella, and P. M. Congedo, "Comparison between wind power prediction models based on wavelet decomposition with Least-Squares Support Vector Machine (LS-SVM) and Artificial Neural Network (ANN)," Energies, vol. 7, no. 8, pp. 5251-5272, 2014.

[24] F. Martínez-Alvarez, A. Troncoso, G. Asencio-Cortés, and J. C. Riquelme, "A survey on data mining techniques applied to electricity-related time series forecasting," Energies, vol. 8, no. 11, pp. 13162-13193, 2015.

[25] H. Zhu, X. Li, Q. Sun, L. Nie, J. Yao, and G. Zhao, "A power prediction method for photovoltaic power plant based on wavelet decomposition and artificial neural networks," Energies, vol. 9, article 11, 2016.

[26] R. Sousa, L. Aguiar-Conraria, and M. J. Soares, "Carbon financial markets: a time-frequency analysis of $\mathrm{CO}_{2}$ prices," Physica A: Statistical Mechanics and Its Applications, vol. 414, pp. 118-127, 2014.

[27] R. Khalfaoui, M. Boutahar, and H. Boubaker, "Analyzing volatility spillovers and hedging between oil and stock markets: evidence from wavelet analysis," Energy Economics, vol. 49, pp. 540-549, 2015.

[28] S. Bahrami, R.-A. Hooshmand, and M. Parastegari, "Short term electric load forecasting by wavelet transform and grey model improved by PSO (particle swarm optimization) algorithm," Energy, vol. 72, pp. 434-442, 2014.

[29] T. Alexandrov, S. Bianconcini, E. B. Dagum, P. Maass, and T. S. McElroy, "A review of some modern approaches to the problem of trend extraction," Econometric Reviews, vol. 31, no. 6, pp. 593624, 2012

[30] F. Benhmad, "Dynamic cyclical comovements between oil prices and US GDP: a wavelet perspective," Energy Policy, vol. 57, pp. 141-151, 2013.

[31] J. C. Reboredo and M. A. Rivera-Castro, "Wavelet-based evidence of the impact of oil prices on stock returns," International Review of Economics and Finance, vol. 29, pp. 145-176, 2014.

[32] D. B. Percival and A. T. Walden, Wavelet Methods for Time Series Analysis, Cambridge University Press, Cambridge, UK, 2002.

[33] R. Jammazi, "Cross dynamics of oil-stock interactions: a redundant wavelet analysis," Energy, vol. 44, no. 1, pp. 750-777, 2012.

[34] S. S. Khaloo, A. A. Ensafi, and T. Khayamian, "Determination of bismuth and copper using adsorptive stripping voltammetry couple with continuous wavelet transform," Talanta, vol. 71, no. 1, pp. 324-332, 2007.
[35] C. Zhang, C. K. Sun, and B. Liu, "Continuous monitoring of NO in flue gas based on wavelet decomposition," Journal of Environmental Monitoring, vol. 13, no. 4, pp. 991-998, 2011.

[36] X.-Y. Gao, H.-Z. An, and W. Fang, "Research on fluctuation of bivariate correlation of time series based on complex networks theory," Acta Physica Sinica, vol. 61, no. 9, Article ID 098902, 2012.

[37] X.-Y. Gao, H.-Z. An, H.-H. Liu, and Y.-H. Ding, "Analysis on the topological properties of the linkage complex network between crude oil future price and spot price," Acta Physica Sinica, vol. 60, no. 6, Article ID 068902, 2011.

[38] H. Z. An, X. Y. Gao, W. Fang, X. Huang, and Y. H. Ding, "The role of fluctuating modes of autocorrelation in crude oil prices," Physica A: Statistical Mechanics and Its Applications, vol. 393, pp. 382-390, 2014.

[39] C.-B. Li, H. Yang, and T. Komatsuzaki, "Multiscale complex network of protein conformational fluctuations in single-molecule time series," Proceedings of the National Academy of Sciences of the United States of America, vol. 105, no. 2, pp. 536-541, 2008.

[40] R. Wackerbauer, A. Witt, H. Atmanspacher, J. Kurths, and H. Scheingraber, "A comparative classification of complexity measures," Chaos, Solitons and Fractals, vol. 4, no. 1, pp. 133-173, 1994.

[41] S. Dajčman, "Interdependence between some major European stock markets-a wavelet lead/lag analysis," Prague Economic Papers, vol. 22, no. 1, pp. 28-49, 2013.

[42] A. K. Tiwari, C. Oros, and C. T. Albulescu, "Revisiting the inflation-output gap relationship for France using a wavelet transform approach," Economic Modelling, vol. 37, pp. 464-475, 2014.

[43] A. Barrat, M. Barthélemy, R. Pastor-Satorras, and A. Vespignani, "The architecture of complex weighted networks," Proceedings of the National Academy of Sciences of the United States of America, vol. 101, no. 11, pp. 3747-3752, 2004. 


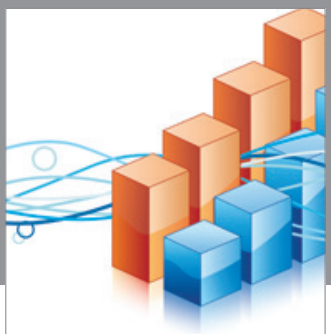

Advances in

Operations Research

vatem alat4

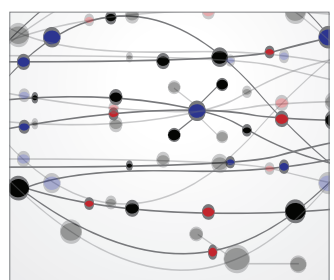

\section{The Scientific} World Journal
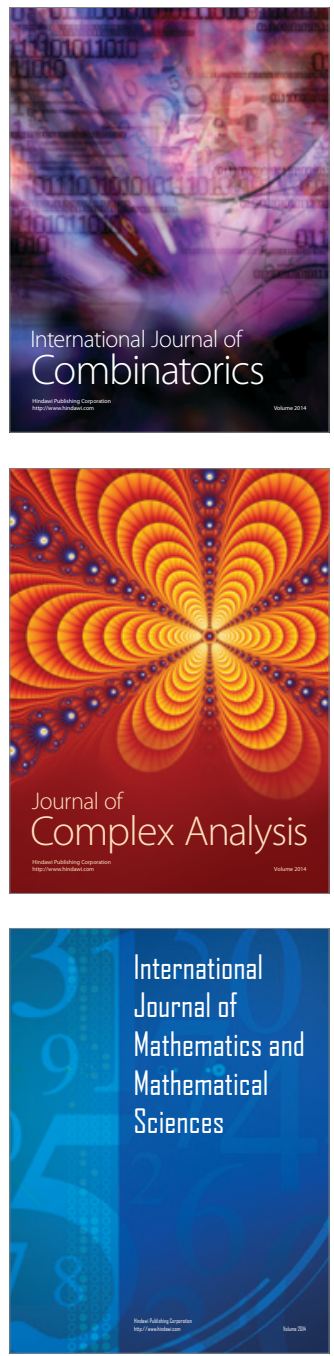
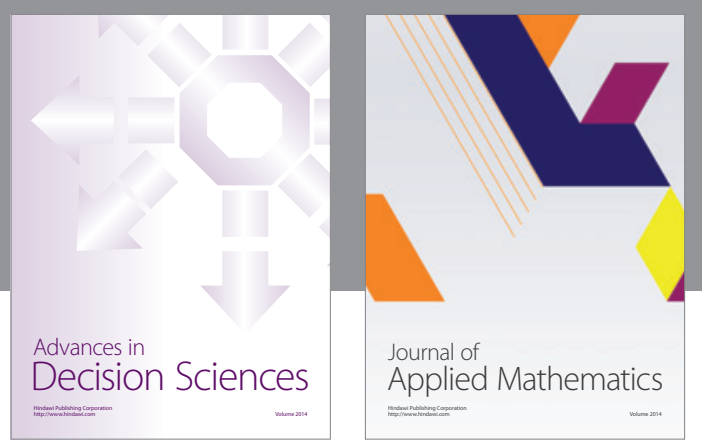

Algebra

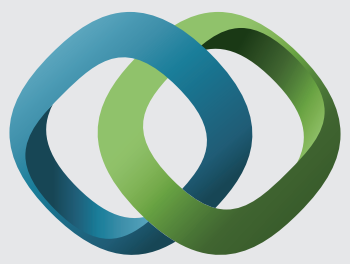

\section{Hindawi}

Submit your manuscripts at

http://www.hindawi.com
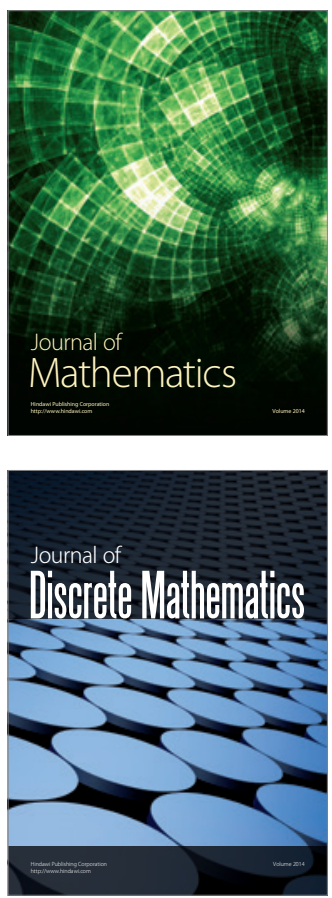

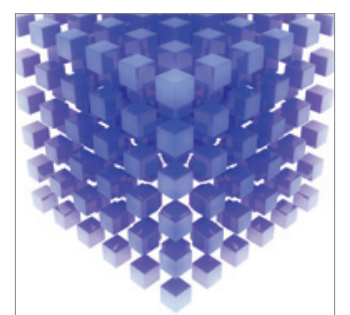

Mathematical Problems in Engineering
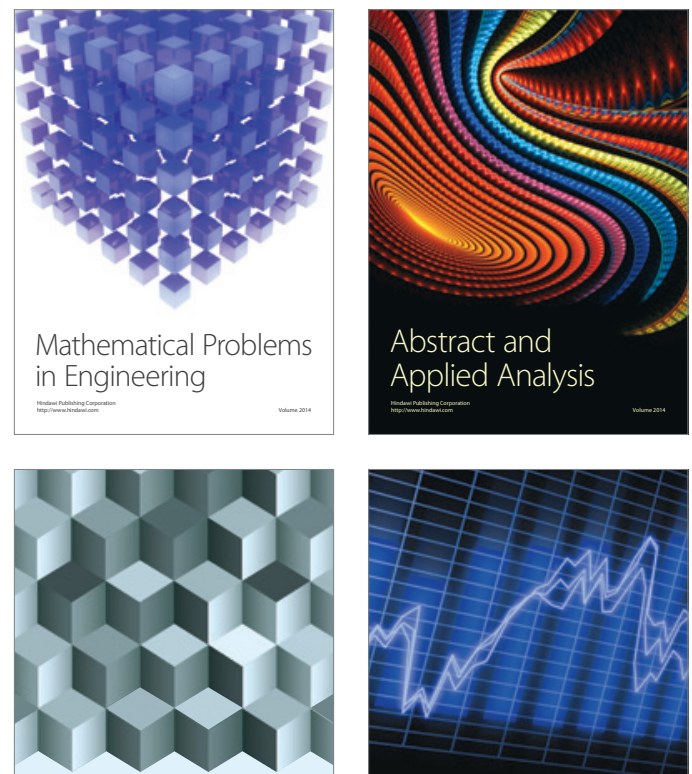

Journal of

Function Spaces

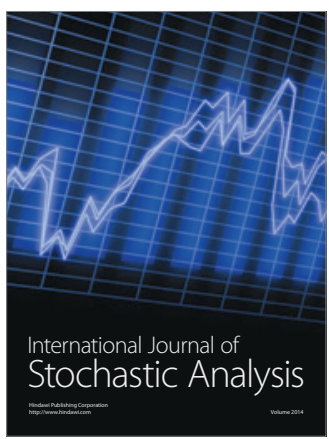

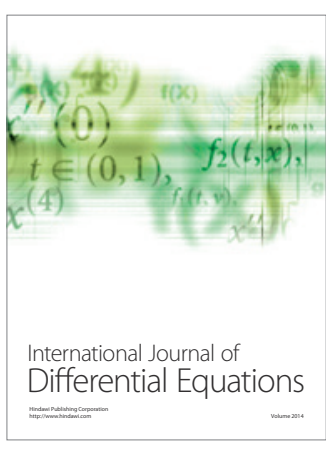
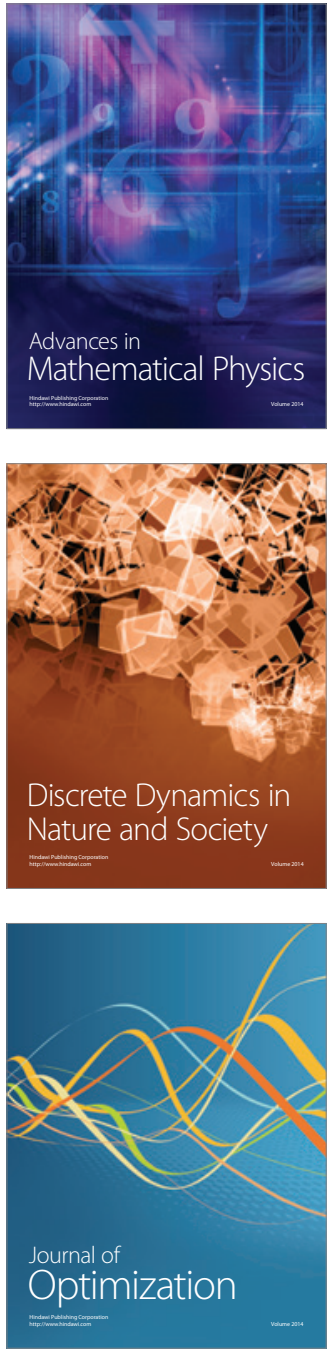